\title{
Qubits from number states and Bell inequalities for number measurements
}

Jan-Åke Larsson

The self-archived postprint version of this journal article is available at Linköping University Institutional Repository (DiVA):

http:// urn.kb.se/ resolve?urn=urn:nbn:se:liu:diva-23110

N.B.: When citing this work, cite the original publication.

Larsson, J., (2003), Qubits from number states and Bell inequalities for number measurements, Physical Review A. Atomic, Molecular, and Optical Physics, 67, 022108-1-022108-8.

https:// doi.org/ 10.1103/ PhysRevA.67.022108

Original publication available at:

https:// doi.org/ 10.1103/PhysRevA.67.022108

Copyright: American Physical Society

http://www.aps.org/ 


\title{
Qubits from number states and Bell inequalities for number measurements
}

\author{
Jan- ̊̊ke Larsson* \\ MaPhySto, Department of Mathematical Sciences, Aarhus University, Ny Munkegade, DK-8000 Aarhus C, Denmark
}

(Received 28 August 2002; published 24 February 2003)

\begin{abstract}
Bell inequalities for number measurements are derived via the observation that the bits of the number indexing a number state are proper qubits. Violations of these inequalities are obtained from the output state of the nondegenerate optical parametric amplifier.
\end{abstract}

DOI: 10.1103/PhysRevA.67.022108

PACS number(s): 03.65.Ud, 03.65.Ta, 03.67.- a

\section{INTRODUCTION}

The Bell inequality [1] and its descendants (see e.g., Ref. [2]) are the main tools used when studying the question whether a bipartite system can be described by a local realist model or not. In such a model, the properties of the system exist independently of measurement, and measurements on one subsystem do not influence the other subsystem. For a proper statement of the exact properties of a local realist model, see conditions (i)-(iii) below. Under the assumption of local realism, a bound on the statistics from a bipartite system can be derived, a Bell inequality, e.g., (10) below. A bipartite quantum system is not described by such a model, and indeed, the mentioned statistical bound is violated by statistics obtained from the singlet spin- $\frac{1}{2}$ quantum state. Thus, that system cannot be described by a local realist model.

The two parts of a bipartite spin- $\frac{1}{2}$ system each have a quantum description that is (complex) two dimensional, and this is appropriate for a violation of the inequalities in Refs. $[1,2]$. There are a number of treatments of higherdimensional systems, e.g., Refs. [3,4], but here an attempt will be made to look at an infinite-dimensional system and its number operator. Some problems that emerge will be discussed and inequalities suitable for the situation will be derived. These inequalities are violated by the state produced in the nondegenerate optical parametric amplifier (NOPA).

The interest in these questions stems from the EinsteinPodolsky-Rosen (EPR) paradox [5]. Perhaps one should comment on the relation between the continuous-variable, infinite-dimensional quantum system used in the original EPR paradox, where position $(x)$ and momentum $(p)$ are used, and the finite-dimensional spin-based approach of Bohm [6]. Bell [7] has presented a local realist model for position and momentum measurements on the original EPR state, constructed using the Wigner function representation of the EPR state as a joint probability of the measurement results. The Wigner function generally has all the properties of a probability measure except one: it can be negative (a proper probability measure is always positive). However, in this particular case the Wigner function is positive, so it can be used as a proper probability measure. One could be led to

\footnotetext{
*Present address: Matematiska Institutionen, Linköpings Universitet, SE-581 83 Linköping, Sweden.

Email address: jalar@mai.liu.se
}

think that this implies that the EPR state can be described by a local realist model, but this is not the case: the important thing to note is the statement "for position and momentum measurements." That is, nothing is said about other measurements; the quantum state contains more than just information about position and/or momentum. In fact, if one instead uses measurements of parity, one can interpret the Wigner function as a correlation function for these parity measurements, and then regularized EPR states are nonlocal [8].

Furthermore, in Ref. [9], pseudospin operators based on parity are introduced, and from them a violation of the Clauser-Horne-Shimony-Holt (CHSH) inequality [2] is derived using the $\mid$ NOPA $\rangle$ state. The steps will be briefly reiterated here as they will be important in what follows. The pseudospin operators are

$$
\begin{aligned}
& s_{z}=\sum_{n=0}^{\infty}(-1)^{n}|n\rangle\langle n|, \\
& s_{+}=\left(s_{-}\right)^{\dagger}=\sum_{n=0}^{\infty}|2 n\rangle\langle 2 n+1|, \\
& s_{x}=s_{+}+s_{-}, \quad s_{y}=-i\left(s_{+}-s_{-}\right),
\end{aligned}
$$

and these satisfy the usual commutation relations

$$
\left[s_{z}, s_{ \pm}\right]= \pm 2 s_{ \pm}, \quad\left[s_{+}, s_{-}\right]=s_{z} .
$$

Please observe the change of sign convention in Eq. (1) from that of Ref. [9]. Now, the pseudospin operator $\hat{\mathbf{s}}$ $=\left(s_{x}, s_{y}, s_{z}\right)$ in a sense corresponds to the spin operator $\hat{\boldsymbol{\sigma}}$ for a normal spin- $\frac{1}{2}$ system. In the general case, a measurement of pseudospin can be made along a certain "direction" a by using the operator $\mathbf{a} \cdot \hat{\mathbf{s}}$, but for our purposes, planar variation is sufficient, so let us use the notation

$$
s_{\theta}=\cos (\theta) s_{z}+\sin (\theta) s_{x} .
$$

The two parts of our bipartite system can be subjected to individual measurements of the above type, and we will use the shorthand notation $s_{\alpha} s_{\beta}^{\prime}$ to denote $s_{\alpha} \otimes s_{\beta}$ below.

In our bipartite system we will use the |NOPA $\rangle$ state as our entangled state

$$
|\mathrm{NOPA}\rangle=\frac{1}{\cosh r} \sum_{n=0}^{\infty} \tanh ^{n} r|n\rangle \otimes|n\rangle .
$$


The parameter $r$ is usually referred to as the squeezing $p a$ rameter, and is a measure of the amount of squeezing in the system. This state is sometimes referred to as a regularized EPR state, and at infinite squeezing $(r \rightarrow \infty)$, the state approaches the idealized state used in the original EPR paper [5]. A simple calculation yields [9]

$$
\begin{aligned}
& \left\langle\mathrm{NOPA}\left|s_{z} s_{z}^{\prime}\right| \mathrm{NOPA}\right\rangle=1, \\
& \left\langle\mathrm{NOPA}\left|s_{x} s_{x}^{\prime}\right| \mathrm{NOPA}\right\rangle=\tanh 2 r=: K .
\end{aligned}
$$

$K$ is a (strictly increasing) function of $r, K=0$ when $r=0$ and $K \rightarrow 1$ when $r \rightarrow \infty$; consequently $K$ can equally well be used as a measure of the amount of squeezing in this state. Finally, we have

$$
\left\langle\mathrm{NOPA}\left|s_{\alpha} s_{\beta}^{\prime}\right| \mathrm{NOPA}\right\rangle=\cos \alpha \cos \beta+K \sin \alpha \sin \beta .
$$

The results of the individual measurements $s_{\alpha} \otimes I$ and $I \otimes s_{\beta}$ will be denoted by $S_{\alpha}$ and $S_{\beta}^{\prime}$. That is, these are the classical \pm 1 values registered from measurement, e.g., written down on a piece of paper or similar. The question is now if these results can be described under the assumption of local realism,

(i) Realism. There is a classical probabilistic model where the results depend on a "hidden variable" $\lambda$, i.e.,

$$
\begin{aligned}
& S_{\alpha}=S_{\alpha}(\lambda), \\
& S_{\beta}^{\prime}=S_{\beta}^{\prime}(\lambda) .
\end{aligned}
$$

(ii) Locality. The model is local, such that measurement settings at one subsystem does not affect the other subsystem, i.e.,

$$
\begin{aligned}
& S_{\alpha}(\lambda) \text { is independent of } \beta, \\
& S_{\beta}^{\prime}(\lambda) \text { is independent of } \alpha .
\end{aligned}
$$

(iii) Result restriction. The measurement results are restricted in size,

$$
\left|S_{\alpha}(\lambda)\right| \leqslant 1, \quad\left|S_{\beta}^{\prime}(\lambda)\right| \leqslant 1 .
$$

When this is the case, we have the CHSH inequality [2]

$$
\left|E\left(S_{\alpha} S_{\gamma}^{\prime}\right)+E\left(S_{\alpha} S_{\delta}^{\prime}\right)\right|+\left|E\left(S_{\beta} S_{\gamma}^{\prime}\right)-E\left(S_{\beta} S_{\delta}^{\prime}\right)\right| \leqslant 2 .
$$

However, using the $\mid$ NOPA $\rangle$ state (in shorthand notation), we have

$$
\begin{aligned}
& \left|\left\langle s_{0} s_{\gamma}^{\prime}\right\rangle+\left\langle s_{0} s_{-\gamma}^{\prime}\right\rangle\right|+\left|\left\langle s_{\pi / 2} s_{\gamma}^{\prime}\right\rangle-\left\langle s_{\pi / 2} s_{-\gamma}^{\prime}\right\rangle\right| \\
& \quad=2(|\cos \gamma|+K|\sin \gamma|) .
\end{aligned}
$$

With e.g., $\gamma=\arctan K$, the maximum of the right-hand side is obtained at $2 \sqrt{1+K^{2}}>2$. The conclusion is that (i) or (ii) (or both) must fail, since (iii) always holds in this setting.

In the ideal case, there is a violation at any nonzero squeezing, but in a noisy setting the violation will be lowered by the noise, so that a certain lowest squeezing will be required. Note that when $K=1$, the violation will be as large as that generated by the singlet state in the original setting. This corresponds to an infinite squeezing parameter $r$, i.e., the original EPR state [5], but unfortunately infinite squeezing cannot be achieved in practice. Note that the angle $\gamma$, at which there is maximum violation, depends on the squeezing.

The detector-efficiency problem [10] is less of an issue here than in the usual Bell inequality because, e.g., lost photons in an optical implementation will not imply that experimental runs are dropped from the statistics. Instead such losses will change the measured parity, introducing noise in the statistics and leading to a situation similar to that of the ion-trap experiment of Rowe et al. [11], where also "dark" events lead to increased noise in the experimental data. Thus, we do not need to use auxiliary assumptions such as the no-enhancement assumption [12]. One of the motivations of Refs. [8,9] and the present paper is to derive a violation of local realism from continuous-variable systems using only the assumptions (i)-(iii) above. A different philosophy is used in some previous proposals for continuous-variable Bell inequalities, where additional assumptions are necessary and in some cases built into the formalism (see e.g., Refs. [1319]). It should be noted that usage of these assumptions usually lead to simpler experimental implementations than is expected from the present treatment.

\section{AN INFINITE COMMUTING HIERARCHY}

To extend this, let us use a different assignment of parity than the usual one. As an example, group the number states two-by-two, assign "even" parity to the number states in the first group and "odd" parity to the next group, and so on. Above, we had

$$
s_{z}=+|0\rangle\langle 0|-| 1\rangle\langle 1|+| 2\rangle\langle 2|-| 3\rangle\langle 3|+\cdots,
$$

but now, a similar expression would be

$$
\begin{aligned}
s_{z, 2}= & +(|0\rangle\langle 0|+| 1\rangle\langle 1|)-(|2\rangle\langle 2|+| 3\rangle\langle 3|) \\
& +(|4\rangle\langle 4|+| 5\rangle\langle 5|)-(|6\rangle\langle 6|+| 7\rangle\langle 7|)+\cdots
\end{aligned}
$$

Adjusting the expressions for $s_{ \pm}$in Eq. (1) for this case, we obtain

$$
\begin{aligned}
& s_{z, 2}=\sum_{n=0}^{\infty}(-1)^{n}(|2 n\rangle\langle 2 n|+| 2 n+1\rangle\langle 2 n+1|), \\
& s_{+, 2}=\left(s_{-, 2}\right)^{\dagger}=\sum_{n=0}^{\infty}[|4 n\rangle\langle 4 n+2|+| 4 n+1\rangle\langle 4 n+3|], \\
& s_{x, 2}=s_{+, 2}+s_{-, 2}, \quad s_{y, 2}=-i\left(s_{+, 2}-s_{-, 2}\right) .
\end{aligned}
$$

Also in this case, it is easy to check that there is a correspondence to a spin- $\frac{1}{2}$ system.

Extending this to arbitrary $d$-by- $d$ grouping is equally simple, 


$$
\begin{aligned}
& s_{z, d}=\sum_{n=0}^{\infty}(-1)^{n} \sum_{k=0}^{d-1}|d n+k\rangle\langle d n+k|, \\
& s_{+, d}=\left(s_{-, d}\right)^{\dagger}=\sum_{n=0}^{\infty} \sum_{k=0}^{d-1}|2 d n+k\rangle\langle 2 d n+k+d|, \\
& s_{x, d}=s_{+, d}+s_{-, d}, \quad s_{y, d}=-i\left(s_{+, d}-s_{-, d}\right) .
\end{aligned}
$$

For example,

$$
\begin{aligned}
s_{z, 3}= & +(|0\rangle\langle 0|+| 1\rangle\langle 1|+| 2\rangle\langle 2|) \\
& -(|3\rangle\langle 3|+| 4\rangle\langle 4|+| 5\rangle\langle 5|) \\
& +(|6\rangle\langle 6|+| 7\rangle\langle 7|+| 8\rangle\langle 8|)+\cdots,
\end{aligned}
$$

while the $d=3$ spin step would be

$$
\begin{aligned}
s_{+, 3}= & |0\rangle\langle 3|+| 1\rangle\langle 4|+| 2\rangle\langle 5| \\
& +|6\rangle\langle 9|+| 7\rangle\langle 10|+| 8\rangle\langle 11|+\cdots
\end{aligned}
$$

Another simple calculation yields

$$
\begin{aligned}
& \left\langle\mathrm{NOPA}\left|s_{z, d} s_{z, d}^{\prime}\right| \mathrm{NOPA}\right\rangle=1, \\
& \left\langle\mathrm{NOPA}\left|s_{x, d} s_{x, d}^{\prime}\right| \mathrm{NOPA}\right\rangle=\frac{2 \tanh ^{d} r}{1+\tanh ^{2 d} r}=: K_{d} .
\end{aligned}
$$

Note that if $0<r<\infty, K_{d}$ decreases when $d$ increases. In a similar fashion as before,

$$
\left\langle\mathrm{NOPA}\left|s_{\alpha, d} s_{\beta, d}^{\prime}\right| \mathrm{NOPA}\right\rangle=\cos \alpha \cos \beta+K_{d} \sin \alpha \sin \beta .
$$

With the same notational conventions as above, we have under local realism [(i)-(iii)], that

$$
\begin{aligned}
& \left|E\left(S_{\alpha, d} S_{\gamma, d}^{\prime}\right)+E\left(S_{\alpha, d} S_{\delta, d}^{\prime}\right)\right| \\
& \quad+\left|E\left(S_{\beta, d} S_{\gamma, d}^{\prime}\right)-E\left(S_{\beta, d} S_{\delta, d}^{\prime}\right)\right| \leqslant 2,
\end{aligned}
$$

and similarly to Eq. (11),

$$
\begin{aligned}
& \left|\left\langle s_{0, d} s_{\gamma, d}^{\prime}\right\rangle+\left\langle s_{0, d} s_{-\gamma, d}^{\prime}\right\rangle\right|+\left|\left\langle s_{\pi / 2, d} s_{\gamma, d}^{\prime}\right\rangle-\left\langle s_{\pi / 2, d} s_{-\gamma, d}^{\prime}\right\rangle\right| \\
& \quad=2\left(|\cos \gamma|+K_{d}|\sin \gamma|\right) .
\end{aligned}
$$

Again for nonzero squeezing a violation of Ineq. (20) is obtained at any positive $d$, but it decreases as $d$ grows. Here, maximum-violation $\gamma$ depends both on the squeezing and on the $d$.

It is not generally true that the pseudospin operators commute for different $d$. For example, the operators for $d=2$ and $d=3$ do not commute; while it is true that $\left[s_{z, 2}, s_{z, 3}\right]=0$, we have $\left[s_{x, 2}, s_{x, 3}\right] \neq 0$. However, an important case when they do commute is when the $d$ 's are related by multiplication of an even number. This is easily verified by inspection in the relation between $d=1$ as in Eq. (1) and $d^{\prime}=2$ as in Eq. (14), and can also be extended to any situation where $d^{\prime}=2^{k} d$; the spin operators $s_{ \pm, d}$ then only exchange number states entirely within the groups defined by $s_{z, d^{\prime}}$ and $s_{ \pm, d^{\prime}}$, and $s_{z, d}$ performs the same sign change within each such group. We obtain an infinite commuting hierarchy of spin systems,

$$
\left\{s_{z, 2^{k}, s_{-, 2^{k}}}=\left(s_{+, 2^{k}}\right)^{\dagger}\right\}_{k \in \mathbb{N}} \text {. }
$$

Using the bipartite $\mid$ NOPA $\rangle$ state, there is a simultaneous, separate violation of a Bell inequality within each spin system in this hierarchy.

\section{NEW NUMBER OPERATORS?}

Interestingly, the $s_{z, 2^{k}}$ operator corresponds to measuement of bits in the binary representation of $n$, mapping a bit-value 0 into the parity value +1 and a bit-value 1 into parity -1 . This is readily seen in Eqs. (12) and (13) and can be shown generally using Eq. (15). The correspondence is

$$
s_{z, 2^{k}}=1-2 b_{k}
$$

wherein $b_{k}$ is the $k$ th bit of $n$. Obtaining number from parity is also possible,

$$
n=\sum_{k=0}^{\infty} 2^{k} b_{k}=\sum_{k=0}^{\infty} 2^{k} \frac{1-s_{z, 2^{k}}}{2} .
$$

Here, it is very important that the different $s_{z, 2^{k}}$ commute. The above construction is, more or less, the observation that the bits of the number representation are qubits in the usual sense of the word, and we can see above that in the |NOPA $\rangle$ state, there is indeed entanglement of the qubits in the number representation.

The bit correspondence of the $s_{z, 2^{k}}$ operator to the number operator $n$ (henceforth referred to as $n_{z}$ ) hints at similar constructions of number operators $n_{x}$ and $n_{y}$ corresponding to $s_{x, 2^{k}}$ and $s_{y, 2^{k}}$, for example,

$$
n_{x}=\sum_{k=0}^{\infty} 2^{k} \frac{1-s_{x, 2^{k}}}{2} .
$$

Unfortunately, this construction is problematic, for example, the eigenstates of $n_{x}$ and $n_{y}$ will not be normal states; let us determine $\left|0_{x}\right\rangle$. All the $n_{x}$ bits are zero which corresponds to the eigenvalue +1 of all $s_{x, 2^{k}}$. From Eq. (1) an eigenstate $|\psi\rangle$ of $s_{x, 1}$ with the eigenvalue +1 will have the property

$$
\left\langle 0_{z} \mid \psi\right\rangle=\left\langle 1_{z} \mid \psi\right\rangle
$$

and similarly, from Ref. (14) an eigenstate $|\varphi\rangle$ of $s_{x, 2}$ with the eigenvalue +1 will follow

$$
\begin{aligned}
& \left\langle 0_{z} \mid \varphi\right\rangle=\left\langle 2_{z} \mid \varphi\right\rangle, \\
& \left\langle 1_{z} \mid \varphi\right\rangle=\left\langle 3_{z} \mid \varphi\right\rangle .
\end{aligned}
$$

Continuing (infinitely), a simultaneous eigenstate of $s_{x, 2^{k}}$ with the eigenvalue +1 for all $k$ 's will have all coefficients equal in the $n_{z}$ basis, i.e., of the form 


$$
C \sum_{n=0}^{\infty}\left|n_{z}\right\rangle
$$

This is not a normal state; it has infinite energy. Nevertheless, it has a bit value of 0 at all positions when measuring $n_{x}$, so choosing $\left|0_{x}\right\rangle$ so that $\left\langle 0_{z} \mid 0_{x}\right\rangle=1$, we have (with a certain abuse of notation [21]

$$
\left|0_{x}\right\rangle=\sum_{n=0}^{\infty}\left|n_{z}\right\rangle
$$

More generally, eigenstates to the eigenvalues \pm 1 of the spin operator $s_{x, d}[$ see Eq. (15)] have the properties

$$
\begin{gathered}
\left\langle 0_{z} \mid \varphi\right\rangle= \pm\left\langle d_{z} \mid \varphi\right\rangle, \\
\left\langle 1_{z} \mid \varphi\right\rangle= \pm\left\langle(d+1)_{z} \mid \varphi\right\rangle, \\
\vdots \\
\left\langle(d-1)_{z} \mid \varphi\right\rangle= \pm\left\langle(2 d-1)_{z} \mid \varphi\right\rangle,
\end{gathered}
$$

where the eigenvalue in question decides what sign the righthand sides have. The vector $\left|m_{x}\right\rangle$ is a simultaneous eigenvector to all $s_{x, 2^{k}}$, and by choosing it so that

$$
\left\langle 0_{z} \mid m_{x}\right\rangle=1
$$

we obtain [put $d=2^{k}$ in Eq. (30)]

$$
\begin{aligned}
&\left\langle\left(2^{k}\right)_{z} \mid m_{x}\right\rangle= \pm 1 \\
&\left\langle\left(2^{k}+1\right)_{z} \mid m_{x}\right\rangle=\left\langle\left(2^{k}\right)_{z} \mid m_{x}\right\rangle\left\langle 1_{z} \mid m_{x}\right\rangle, \\
& \vdots \\
&\left\langle\left(2^{k+1}-1\right)_{z} \mid m_{x}\right\rangle=\left\langle\left(2^{k}\right)_{z} \mid m_{x}\right\rangle\left\langle\left(2^{k}-1\right)_{z} \mid m_{x}\right\rangle .
\end{aligned}
$$

To determine the sign above, first note that

$$
\left\langle 1_{z} \mid m_{x}\right\rangle=\left\{\begin{array}{lll}
+1, & m \text { even } \\
-1, & m \text { odd }
\end{array}\right.
$$

This is because $\left|m_{x}\right\rangle$ is an eigenvector to $s_{x, 1}$ with eigenvalue +1 if the lowest bit of $m$ is zero ( $m$ is even), and with eigenvalue -1 if the lowest bit of $m$ is one ( $m$ is odd). In other words, $\left\langle 1_{z} \mid m_{x}\right\rangle=(-1)^{m \wedge 1}$, where $\wedge$ denotes the bitwise AND operation of the two numbers. Continuing, we have

$$
\left\langle\left(2^{k}\right)_{z} \mid m_{x}\right\rangle= \begin{cases}+1, & m \wedge 2^{k}=0 \\ -1, & m \wedge 2^{k}=2^{k}\end{cases}
$$

And finally, expanding $n$ binary,

$$
\begin{aligned}
\left\langle n_{z} \mid m_{x}\right\rangle & =\left\langle\left.\left(\sum_{k=1}^{K} b_{k} 2^{k}\right)\right|_{z}\right\rangle \\
& =\left\langle\left(b_{K} 2^{K}\right)_{z} \mid m_{x}\right\rangle\left\langle\left(\sum_{k=1}^{K-1} b_{k} 2^{k}\right)_{z} \mid m_{x}\right\rangle \\
& =\cdots=\prod_{k=1}^{K}\left\langle\left(b_{k} 2^{k}\right)_{z} \mid m_{x}\right\rangle .
\end{aligned}
$$

In the right-hand side above, there will be a -1 factor each time $m \wedge b_{k} 2^{k}$ is nonzero. By simply counting the number of bits that are set in $m \wedge n$ [and denoting the result $\mathcal{N}(m \wedge n)]$, we arrive at

$$
\left|m_{x}\right\rangle=\sum_{n=0}^{\infty}(-1)^{\mathcal{N}(m \wedge n)}\left|n_{z}\right\rangle
$$

In the same manner, one can deduce

$$
\left|m_{y}\right\rangle=\sum_{n=0}^{\infty}(-1)^{\mathcal{N}(m \wedge n)} i^{\mathcal{N}(n)}\left|n_{z}\right\rangle
$$

The connection between the eigenstates of $n_{x}$ and $n_{y}$ is more problematic, since all of these are non-normal, and this relation will not be reproduced here [21].

Unfortunately, the non-normal eigenstates of $n_{x}$ implies that when measuring $n_{x}$ the state would be projected onto a non-normal state (for a finite measurement result, using von Neumann measurement theory). Such a measurement would generate an infinite-energy output quantum state, which seems to be a serious deficit in this approach.

Conversely, consider a measurement of $n_{x}$ on the vacuum. The state $\left|0_{z}\right\rangle$ (the vacuum) is an eigenstate to the original number operator $n_{z}$ that has zeros at all bit values, corresponding to the eigenvalue +1 of $s_{z, 2^{k}}$ for all $k$ 's. The properties of a spin system tell us that it is to be expected that a measurement of any $s_{x, 2^{k}}$ will yield equally probable results \pm 1 . Thus, the bits of $n_{x}$ will be evenly distributed, and the probability of getting a finite result from a measurement of $n_{x}$ on the vacuum is zero.

This is actually true for any finite-energy state $|\psi\rangle$ : as $k$ grows in $s_{z, 2^{k}}$, we are looking at higher and higher bits of $n_{z}$. Since the state has finite energy, the probability of a bit being set will tend to zero as $k$ grows, and equivalently the probability of the result +1 when measuring $s_{z, 2^{k}}$ will tend to zero as $k$ grows. This in turn means that the probability of the result +1 when measuring $s_{x, 2^{k}}$ will tend to $\frac{1}{2}$, and equivalently that the probability of bit $k$ being unset in a measurement of $n_{x}$ will tend to $\frac{1}{2}$. The probability may never reach $\frac{1}{2}$, but we know that whenever $k$ exceeds some (large) $K$, this probability will be less than $\frac{2}{3}$. Now, the probability of getting a result bounded by $2^{k}$ is

$$
\left\langle\psi\left|\chi_{n_{x} \leqslant 2^{k}}\right| \psi\right\rangle<\prod_{l=k+1}^{k+L}\left\langle\psi\left|\chi_{n_{x} \wedge 2^{l}=0}\right| \psi\right\rangle<\left(\frac{2}{3}\right)^{L} \rightarrow 0, \quad L \rightarrow \infty,
$$


Here, $\chi_{A}$ denotes the projector onto the subspace where the property $A$ holds. Inequality (38) is valid for any $k$ (larger than $K$ ) and thus, for any finite-energy state, measurement of $n_{x}$ (almost) never yields a finite result.

The above-mentioned problems are two sides of the same coin: if a measurement of $n_{x}$ yields a finite value, the output state from the measurement process has infinite energy; conversely, if a finite-energy state is input in to a measurement of $n_{x}$, the result is always infinite. It does seem very problematic to construct a Bell inequality for this type of number measurements. In fact, the original number operator itself is an unbounded operator and we want to derive a statistical bound for it (our desired Bell inequality). One way around these problems is to truncate the operators at the $d$ th bit, and we will use, e.g.,

$$
n_{z, d}=\sum_{k=0}^{d-1} 2^{k} \frac{1-s_{z, 2^{k}}}{2} .
$$

These operators all yield finite measurement results and finite-energy output states (with a finite-energy input state), and enables the construction of a Bell inequality.

\section{A BELL INEQUALITY FOR NUMBER MEASUREMENTS}

We have now constructed an infinite commuting hierarchy of pseudospin systems, each violating a Bell inequality. We have also established that the $s_{z, 2^{k}}$ operator of each pseudospin system corresponds to one of the bits of the number operator $n\left(=n_{z}\right)$, and more interestingly, each such bit is a qubit in the standard sense of the word. Measurement results can now be represented equally well in the language of spin $( \pm 1)$ as in the language of bits $\left[\frac{1}{2}-\left( \pm \frac{1}{2}\right)\right]$, and we should be able to rewrite our Bell inequalities (10) and (20) in the language of bits rather than spin.

To complete this rewrite, one question remains: we multiply spin-measurement results $\left(S S^{\prime}\right)$ in our Bell inequalities but what function $f\left(B, B^{\prime}\right)$ does this correspond to in the bit language? Listing classical bit values, the corresponding pseudospin values, their product, and the final corresponding bit value in a table, we get

\begin{tabular}{cccc|cc}
$B$ & $B^{\prime}$ & $S$ & $S^{\prime}$ & $S S^{\prime}$ & $f\left(B, B^{\prime}\right)$ \\
\hline 0 & 0 & +1 & +1 & +1 & 0 \\
0 & 1 & +1 & -1 & -1 & 1 \\
1 & 0 & -1 & +1 & -1 & 1 \\
1 & 1 & -1 & -1 & +1 & 0
\end{tabular}

Interestingly, $f$ proves to be exclusive-or (XOR, $\bar{V}$; our sign convention was useful here). This is natural, since the multiplication of our spin values is conveniently interpreted as a test whether the spin values are equal or not; in bit language, such a test is provided by XOR. Moreover,

$$
S S^{\prime}=(1-2 B)\left(1-2 B^{\prime}\right)=1-2 B \bar{\bigvee} B^{\prime} .
$$

Under conditions (i)-(iii) of local realism, and using

$$
B_{\theta, k}=\frac{1}{2}\left(1-S_{\theta, 2^{k}}\right),
$$

we obtain the following directly from Eq. (20):

$$
\begin{aligned}
\mid E\left(B_{\alpha, k}\right. & \left.\bar{\bigvee} B_{\gamma, k}^{\prime}\right)+E\left(B_{\alpha, k} \bar{\vee} B_{\delta, k}^{\prime}\right)-1 \mid \\
& +\left|E\left(B_{\beta, k} \bar{\vee} B_{\gamma, k}^{\prime}\right)-E\left(B_{\beta, k} \bar{\bigvee} B_{\delta, k}^{\prime}\right)\right| \\
= & \frac{1}{2}\left|E\left(S_{\alpha, 2^{k}} S_{\gamma, 2^{k}}^{\prime}\right)+E\left(S_{\alpha, 2^{k}} S_{\delta, 2^{k}}^{\prime}\right)\right| \\
& +\frac{1}{2}\left|E\left(S_{\beta, 2^{k}} S_{\gamma, 2^{k}}^{\prime}\right)-E\left(S_{\beta, 2^{k}} S_{\delta, 2^{k}}^{\prime}\right)\right| \leqslant 1 .
\end{aligned}
$$

A corollary is the more familiar-looking inequality,

$$
\begin{aligned}
& \left|E\left(B_{\alpha, k} \bar{\bigvee} B_{\gamma, k}^{\prime}\right)+E\left(B_{\alpha, k} \bar{\bigvee} B_{\delta, k}^{\prime}\right)\right| \\
& \quad+\left|E\left(B_{\beta, k} \bar{\bigvee} B_{\gamma, k}^{\prime}\right)-E\left(B_{\beta, k} \bar{\bigvee} B_{\delta, k}^{\prime}\right)\right| \leqslant 2 .
\end{aligned}
$$

but Eq. (42) is tighter and will be used below.

We would now like to check for a violation of the above inequality from quantum mechanics, preferably from the previously mentioned $\mid$ NOPA $\rangle$ state. The multiplication of pseudospin operators (quantum-mechanical) translates into a "quantum XOR,"

$$
s s^{\prime}=(1-2 b)\left(1-2 b^{\prime}\right)=1-2 b \bar{\vee} b^{\prime},
$$

so that

$$
b \bar{\vee} b^{\prime}=b+b^{\prime}-2 b b^{\prime} .
$$

This has all the properties expected from an XOR operation, and is a noncommutative operation, e.g.,

$$
\begin{aligned}
b_{x} \bar{\bigvee} b_{z}-b_{z} \bar{\bigvee} b_{x} & =\frac{1-s_{x} s_{z}}{2}-\frac{1-s_{z} s_{x}}{2}=\frac{\left[s_{z}, s_{x}\right]}{2} \\
& =i s_{y}=i\left(1-2 b_{y}\right) .
\end{aligned}
$$

Using the above, it is easy to check that

$$
\begin{aligned}
\left|\left\langle b_{0, d} \bar{V} b_{\gamma, d}^{\prime}\right\rangle+\left\langle b_{0, d} \bar{\vee} b_{-\gamma, d}^{\prime}\right\rangle-1\right| & \\
& +\left|\left\langle b_{\pi / 2, d} \bar{\bigvee} b_{\gamma, d}^{\prime}\right\rangle-\left\langle b_{\pi / 2, d} \bar{\vee} b_{-\gamma, d}^{\prime}\right\rangle\right| \\
= & |\cos \gamma|+K_{d}|\sin \gamma| .
\end{aligned}
$$

We again have a violation, although both the bound and the violation are a factor of $\frac{1}{2}$ less than in Eqs. (20) and (21). This makes the absolute quantum-mechanical violation of Ineq. (42) less than that of Ineq. (20), but the sensitivity to experimental problems is the same; we have simply represented the data differently.

Until now we have treated the pseudospin systems as individual systems, measuring and comparing them one-byone. Let us now do a joint treatment of the first $d$ bits by using the measurement results $N_{z, d}$ and so on, written in binary form, for example,

$$
N_{z, d}=\sum_{k=0}^{d-1} 2^{k} B_{z, k}
$$


The truncation to $d$ bits is useful to avoid the aforementioned problems of the number operators, but will also be necessary to give a useful bound below. We now perform a comparison of the individual bits in each (truncated) number by using bitwise XOR,

$$
N_{\alpha, d} \bar{\vee} N_{\gamma, d}^{\prime}=\left(\sum_{k=0}^{d-1} 2^{k} B_{\alpha, k}\right) \bar{\vee}\left(\sum_{l=0}^{d-1} 2^{l} B_{\gamma, l}^{\prime}\right)=\sum_{k=0}^{d-1} 2^{k}\left(B, k \bar{\vee} B_{\gamma, k}^{\prime}\right)
$$

For each individual bit, we have the Bell inequality (42) violated by the quantum-mechanical expression (47), and joining these together, we have

$$
\begin{aligned}
& \left|E\left(N_{\alpha, d} \bar{\vee} N_{\gamma, d}^{\prime}\right)+E\left(N_{\alpha, d} \bar{\vee} N_{\delta, d}^{\prime}\right)-\left(2^{d}-1\right)\right|+\left|E\left(N_{\beta, d} \bar{\vee} N_{\gamma, d}^{\prime}\right)-E\left(N_{\beta, d} \bar{\vee} N_{\delta, d}^{\prime}\right)\right| \\
& =\left|E\left(\sum_{k=0}^{d-1} 2^{k}\left(B_{\alpha, k} \bar{\vee} B_{\gamma, k}^{\prime}\right)\right)+E\left(\sum_{k=0}^{d-1} 2^{k}\left(B_{\alpha, k} \bar{\vee} B_{\delta, k}^{\prime}\right)\right)-\sum_{k=0}^{d-1} 2^{k}\right|+\left|E\left(\sum_{k=0}^{d-1} 2^{k}\left(B_{\beta, k} \bar{\vee} B_{\gamma, k}^{\prime}\right)\right)-E\left(\sum_{k=0}^{d-1} 2^{k}\left(B_{\beta, k} \bar{\vee} B_{\delta, k}^{\prime}\right)\right)\right| \\
& \leqslant \sum_{k=0}^{d-1} 2^{k}\left[\left|E\left(B_{\alpha, k} \bar{\vee} B_{\gamma, k}^{\prime}\right)+E\left(B_{\alpha, k} \bar{\bigvee} B_{\delta, k}^{\prime}\right)-1\right|+\left|E\left(B_{\beta, k} \bar{\vee} B_{\gamma, k}^{\prime}\right)-E\left(B_{\beta, k} \bar{\bigvee} B_{\delta, k}^{\prime}\right)\right|\right] \leqslant \sum_{k=0}^{d-1} 2^{k}=2^{d}-1
\end{aligned}
$$

Again we have a more familiar-looking corollary

$$
\begin{aligned}
\left|E\left(N_{\alpha, d} \bar{\vee} N_{\gamma, d}^{\prime}\right)+E\left(N_{\alpha, d} \bar{\vee} N_{\delta, d}^{\prime}\right)\right| & \\
& +\left|E\left(N_{\beta, d} \bar{\vee} N_{\gamma, d}^{\prime}\right)-E\left(N_{\beta, d} \bar{\vee} N_{\delta, d}^{\prime}\right)\right| \\
\leqslant & 2\left(2^{d}-1\right),
\end{aligned}
$$

and again the tighter inequality (50) will be retained; it is our desired Bell inequality for number measurements.

A violation of Eq. (50) is obtained using the |NOPA $\rangle$ state, for which

$$
\begin{array}{r}
\left|\left\langle n_{0, d} \bar{\vee} n_{\gamma, d}^{\prime}\right\rangle+\left\langle n_{0, d} \bar{\vee} n_{-\gamma, d}^{\prime}\right\rangle-\left(2^{d}-1\right)\right| \\
+\left|\left\langle n_{\pi / 2, d} \bar{\vee} n_{\gamma, d}^{\prime}\right\rangle-\left\langle n_{\pi / 2, d} \bar{\vee} n_{-\gamma, d}^{\prime}\right\rangle\right| \\
=\left(2^{d}-1\right)|\cos \gamma|+\sum_{k=0}^{d-1} 2^{k} K_{2^{k}}|\sin \gamma|
\end{array}
$$

and with $\gamma$ chosen properly, the maximum violation is obtained as

$$
\sqrt{\left(2^{d}-1\right)^{2}+\left(\sum_{k=0}^{d-1} 2^{k} K_{2^{k}}\right)^{2}}>2^{d}-1
$$

At infinite squeezing $\left(K_{2^{k}}=1\right.$ for all $\left.k\right)$ we have $\sqrt{2}\left(2^{d}\right.$ $-1)$, which gives the largest possible violation of inequality (50) $[22]$.

One reason for using a bitwise operation here instead of the usual multiplication is the above-mentioned correspondence to multiplication of pseudospins, but there is another, perhaps less evident reason. It is clear that for the |NOPA $\rangle$ state, the result of a measurement of $n_{z}$ is the same as that of $n_{z}^{\prime}$ in the ideal case. This implies that the measurement re- sults of the bits $b_{k}$ and $b_{k}^{\prime}$ are also the same. In a noisy environment, the correlation would be high. However, different bits are not correlated in the same manner, e.g., measurement results from $b_{k}$ and $b_{k+1}^{\prime}$ do not enjoy the same degree of correlation. In fact, even measuring on one part of the bipartite $\mid$ NOPA $\rangle$ state, knowledge of the value of one of the bits (of $n_{z}$, say) gives no statistical information on the value of another. When calculating a product, e.g.,

$$
N_{z, 2} N_{z, 2}^{\prime}=B_{0} B_{0}^{\prime}+4 B_{1} B_{1}^{\prime}+2\left(B_{0} B_{1}^{\prime}+B_{1} B_{0}^{\prime}\right)
$$

it is easy to see that there are terms that mix different bits of the two numbers. It is of course possible to derive a Bell inequality for this expression as well, and the first two terms correspond to a weighted bitwise AND, similar to the bitwise XOR used in this paper. Unfortunately, the parenthesis at the end performs a bitwise AND on different bits of the two numbers. Thus, the last parenthesis yields no usable information, but only additional noise. It would be possible to get around the noise addition by using a state in which knowledge of one bit implies knowledge of another, but this will in effect reduce the available dimensionality of the system; we would, in bit notation, want to use a state close to $|00\rangle \otimes|00\rangle$ $+|11\rangle \otimes|11\rangle$. We can conclude that the bitwise XOR used here is a better choice of "multiplication" in this setting.

Above we used one natural weighting of the bits, while in information-theoretic considerations often another is natural, namely, equal weighting of the bits for which we obtain

$$
\begin{aligned}
& \left|E\left(\mathcal{N}\left(N_{\alpha, d} \bar{\vee} N_{\gamma, d}^{\prime}\right)\right)+E\left(\mathcal{N}\left(N_{\alpha, d} \bar{\vee} N_{\delta, d}^{\prime}\right)\right)-d\right| \\
& \quad+\left|E\left(\mathcal{N}\left(N_{\beta, d} \bar{\vee} N_{\gamma, d}^{\prime}\right)\right)-E\left(\mathcal{N}\left(N_{\beta, d} \bar{\vee} N_{\delta, d}^{\prime}\right)\right)\right| \leqslant d,
\end{aligned}
$$


where $\mathcal{N}\left(N \bar{\nabla} N^{\prime}\right)$ is the number of bits set in the bitwise XOR of $N$ and $N^{\prime}$. Again, using the $\mid$ NOPA $\rangle$ state,

$$
\begin{aligned}
\left|\left\langle\mathcal{N}\left(n_{0, d} \bar{\vee} n_{\gamma, d}^{\prime}\right)\right\rangle+\left\langle\mathcal{N}\left(n_{0, d} \bar{\vee} n_{-\gamma, d}^{\prime}\right)\right\rangle-d\right| \\
\quad+\left|\left\langle\mathcal{N}\left(n_{\pi / 2, d} \bar{\vee} n_{\gamma, d}^{\prime}\right)\right\rangle-\left\langle\mathcal{N}\left(n_{\pi / 2, d} \bar{\vee} n_{-\gamma, d}^{\prime}\right)\right\rangle\right| \\
=d|\cos \gamma|+\sum_{k=0}^{d-1} K_{2^{k}}|\sin \gamma|
\end{aligned}
$$

and the maximum is obtained as

$$
\sqrt{d^{2}+\left(\sum_{k=0}^{d-1} K_{2^{k}}\right)^{2}}>d .
$$

At infinite squeezing, the largest possible violation is obtained at $\sqrt{2} d$.

\section{CONCLUSIONS}

The present paper provides Bell inequalities for number measurements via the observation that the bits of the number operator are true and proper qubits. It extends [9] wherein the authors use the parity pseudospin system corresponding to the lowest qubit of the number operator; here we use a commuting hierarchy of similar systems corresponding to all qubits of $n$. One benefit of this is that the the available entanglement in the $\mid$ NOPA $\rangle$ state is better used. Furthermore, by this construction, continuous-variable systems can be used to violate the Bell inequality via number measurements. Also, via the mentioned qubits, continuous-variable systems are possible to use for quantum-computational tasks.

Unfortunately, on the experimental side, no good method of measuring parity is known, much less the extended parity operators $s_{x, d}, s_{y, d}$, and $s_{z, d}$, or the new (truncated) number operators $n_{x, d}$ and $n_{y, d}$ introduced here. There is an experimentally challenging proposal in Ref. [9] which has not yet been realized. Nevertheless, perhaps Refs. [8,9] and this paper will provide motivation to search for a good measurement procedure.

Another use of this formalism is to extract distinguishable bipartite entangled spin systems (or rather, pseudospin systems) out of a bipartite entangled system, where each part consists of undistinguishable pieces [20]. Measuring the number of pieces (atoms or whatnot) that have a certain property instead of identifying exactly which pieces (atoms) that have the property does seem simpler to achieve. But there are still the experimental challenges noted above, of course. And it takes $2^{k}$ undistinguishable pieces of each part of the system to establish $k$ distinguishable spin systems, which can make the procedure comparatively costly.

Inequalities (10), (20), (42), (50), and (55) are all examples of choices of different weighting of the bits (or, equivalently, the parity pseudospins). In fact, any weighting one finds reasonable can be used, for example, to adapt for the case where the lowest bits are not really accessible, as may happen in the experimental setup of Ref. [20]. This is one way of deriving more inequalities from the above approach, and another is to allow the angles to differ for different bits. We would then obtain a larger violation from a finitely squeezed $\mid$ NOPA $\rangle$ state than the one shown above, because the best angles vary from one $d$ to another in the Bell inequality (20). A third extension is to use qutrits (spin-1 correspondence) instead of qubits in the approach, or indeed so-called quNits $[\operatorname{spin}-(N-1) / 2$ correspondence] for arbitrary $N$, together with an inequality more suited to such a situation $[3,4]$.

As to noise sensitivity and other experimental problems, previous results are of course usable in a bitwise analysis as indicated above. But since an error may affect several bits in the number-measurement approach, a more detailed analysis is necessary. Further analysis is perhaps of limited value until a good proposal for measuring $n_{x}$ and $n_{y}$ is available, wherein the properties of possible experimental problems are better visible.

Finally, this is certainly not the only approach to obtain Bell inequalities for continuous-variable systems, even when taking into account the possible extensions mentioned above. But the present treatment is a step towards understanding the difficult and interesting issues at hand.

\section{ACKNOWLEDGMENTS}

The author would like to thank E. Polzik and K. Mølmer for valuable discussions. This work has been supported by the Wenner-Gren Foundation and the Royal Swedish Academy of Science. MaPhySto, The Center for Mathematical Physics and Stochastics, is funded by the Danish National Research Foundation.
[1] J.S. Bell, Physics (Long Island City, N.Y.) 1, 195 (1964).

[2] J.F. Clauser, M.A. Horne, A. Shimony, and R.A. Holt, Phys. Rev. Lett. 23, 880 (1969).

[3] D. Kaszlikowski, P. Gnaciński, M. Żukowski, W. Miklaszewski, and A. Zeilinger, Phys. Rev. Lett. 85, 4418 (2000).

[4] D. Collins, N. Gisin, N. Linden, S. Massar, and S. Popescu, Phys. Rev. Lett. 88, 040404 (2002).

[5] A. Einstein, B. Podolsky, and N. Rosen, Phys. Rev. 47, 777 (1935).

[6] D. Bohm, Quantum Theory (Prentice-Hall, New York, 1951).

[7] J.S. Bell, Ann. N.Y. Acad. Sci. 480, 263 (1986).
[8] K. Banaszek and K. Wódkiewicz, Phys. Rev. A 58, 4345 (1998).

[9] Z.-B. Chen, J.-W. Pan, G. Hou, and Y.-D. Zhang, Phys. Rev. Lett. 88, 040406 (2002).

[10] J.-Å. Larsson, Phys. Rev. A 57, 3304 (1998).

[11] M.A. Rowe, D. Kielpinski, V. Meyer, C.A. Scakett, W.M. Itano, C. Monroe, and D.J. Wineland, Nature (London) 409, 791 (2001).

[12] J.F. Clauser and M.A. Horne, Phys. Rev. D 10, 526 (1974).

[13] P. Grangier, M.J. Potasek, and B. Yurke, Phys. Rev. A 38, 3132 (1988). 
[14] S.M. Tan, D.F. Walls, and M.J. Collett, Phys. Rev. Lett. 66, 252 (1991).

[15] E. Santos, Phys. Rev. Lett. 68, 894 (1992).

[16] W.J. Munro and M.D. Reid, Phys. Rev. A 47, 4412 (1993).

[17] B. Yurke and D. Stoler, Phys. Rev. Lett. 79, 4941 (1997).

[18] T.C. Ralph, W.J. Munro, and R.E.S. Polkinghorne, Phys. Rev. Lett. 85, 2035 (2000).

[19] E.H. Huntington and T.C. Ralph, Phys. Rev. A 65, 012306 (2002).

[20] B. Julsgaard, A. Kozhekin, and E.S. Polzik, Nature (London)
413, 400 (2001).

[21] In a situation like this, one should really deal with states in the form of functionals on operators and weak convergence, but perhaps this would lead us astray in our search for a Bell inequality.

[22] A technical note is that the first inequality of (50) cannot be violated by quantum mechanics; while the triangel inequality is used there too (it is also the basis of the Bell inequality), it is used to split commuting measurements rather than noncommuting ones. 